\title{
The follow-up observations for the transit events of WASP-12b
}

\author{
Xiao-bin Wang ${ }^{1}$, Andrew Collier Cameron ${ }^{2}$, Sheng-hong Gu ${ }^{1}$, \\ Xiang-song Fang ${ }^{1}$ and Dong-tao $\mathrm{Cao}^{1}$ \\ ${ }^{1}$ National Astronomical Observatories/Yunnan Observatory, CAS, Kunming, China \\ email: wangxb@ynao.ac.cn \\ ${ }^{2}$ University of St. Andrews, Fife KY16 9SS, UK
}

\begin{abstract}
The four new transit light curves of WASP-12b are analyzed by using MCMC simulation so as to derive the system parameters. If the apsidal precession exists in the orbit of WASP-12b, according to the theory of Gimenez \& Bastero (1995), the rate of precession is estimated as 0.0076 degrees per cycle in the case of orbit with an inclination of $90^{\circ}$.
\end{abstract}

Keywords. planetary systems, celestial mechanics, eclipses

\section{Introduction}

The follow-up observations with high precision for known transiting systems not only can be used to refine planetary parameters, but also to identify the variation of some parameters. Many reasons can result in the variation of parameters. For example, the quadrupole of moment of star, planet and undetectable other planets may produce the precession of line of nodes and apsidal line (Miralda-Escudé 2002). The precession of line of nodes might induce small changes in the shape of transiting light curve, whereas the apsidal precession changes the shape of radial velocity curve. For a very closed-in hot Jupiter $(\mathrm{a}<0.025 \mathrm{AU})$, the quadrupole of moment of planetary tidal bulge is a main source of apsidal precession (Ragozzine \& Wolf 2009). The rate of apsidal precession can be identified in case of orbit with a non-zero eccentricity.

The hot Jupiter WASP-12b discovered by Hebb et al. (2009), is close to its host star $(\mathrm{a}=0.0229 \mathrm{AU})$. The apparent eccentricity is identified by some authors (Hebb et al. 2009; Campo et al. 2011; Lopez-Morales et al. 2010; Husnoo et al. 2011). Thus, WASP-12b is an important candidate to measure the apsidal precession.

\section{Observations and data reduction}

Four transit events of WASP-12b were observed with $1 \mathrm{~m}$ telescope $\left(2 \mathrm{~K}^{*} 2 \mathrm{~K}\right.$ CCD and R-filter) in Kunming, China on Nov. 9, Dec. 14, 15, 2009 and 2.4m telescope (1340*1300 CCD and R-filter) in Lijiang, China on Mar. 18, 2010. The magnitude of selected objects in CCD frames is measured with APPHOT task of IRAF. 26 and 24 "reference stars" for $1 \mathrm{~m}$ and $2.4 \mathrm{~m}$ observations, respectively, are used to simulate systematic errors in photometric data with the methods of Collier Cameron et al. (2006) and Tamuz et al. (2005). Then these simulated systematic effects are removed from the WASP-12b's photometric data. 
Table 1. Part parameters of WASP-12b.

\begin{tabular}{ll}
\hline Sidereal period & $1.091412^{+-8.77 E-06}-1.54 E-05$ days \\
$\mathrm{e}$ & $0.065^{+-0.021}-0.022$ \\
\hline
\end{tabular}

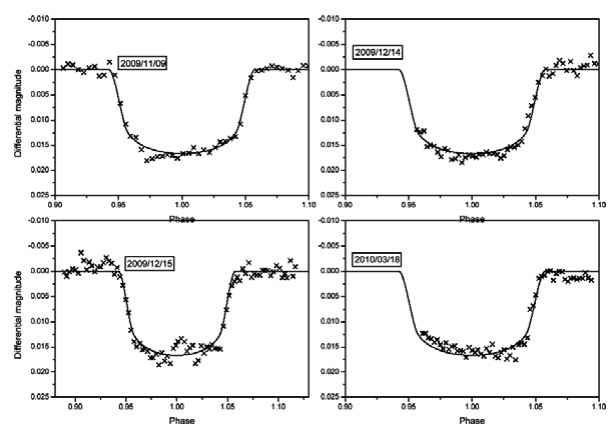

Figure 1. Binned light curves of WASP-12b and relative fitting.

\section{Light curve analysis and conclusions}

The MCMC (Markov Chain Monte Carlo) routine (Collier Cameron et al. 2007) is used to estimate the planetary parameters based on 4 new transit light curves. Firstly, we only analyze 4 new transit light curves of WASP-12b by using the MCMC method. The derived transit period of 1.091437 days is slightly longer than that of Hebb et al. (2009). Fig. 1 shows 4 transit light curves and the model data calculated with the bestfitting parameters. Secondly, the observed light curves combined with the radial velocity data of Hebb et al. (2009) and Husnoo et al. (2011) are analyzed together with MCMC procedure. Table 1 lists the part results. The period of 1.091412 days is different from above one, but is close to the value of Husnoo et al. (2011).

We think that two different periods (sidereal period and transit period) may imply the apsidal precession exists in WASP-12b's orbit. Providing an inclination of 90 degrees, we estimate the rate of apsidal precession according to the equation (15) of Gimenez \& Bastero (1995) is 0.0076 degrees per sidereal cycle. New accurate photometric and radial velocity observations for WASP-12b are needed for obtaining the more accurate rate of precession.

\section{Acknowledgments}

This work is supported by NSFC under grant No.10873031 and Chinese Academy of Sciences under grant KJCX2-YW-T24.

\section{References}

Campo, C. J., Harrington, J., Hardy, R. A., et al. 2011, ApJ, 727, id.125

Collier Cameron, A., et al. 2006, MNRAS, 373, 799

Collier Cameron, A., et al. 2007, MNRAS, 380, 1230

Gimenez, A. \& Bastero, M. 1995, ApJ, 659, 1661

Hebb, L., Collier-Cameron, A., Loeillet, B., et al. 2009, ApJ, 693, 1920

Husnoo, N., Pont, F., \& Hebrard, G., et al. 2011, 413, 2500

Lopez-Morales, M., Coughlin, J. L., Sing, D. K., et al. 2010, ApJ, 716, 36

Miralda-Escude, J. 2002, ApJ, 564, 1019

Ragozzine, D. \& Wolf, A. S. 2009, ApJ, 698, 1778

Tamuz, O., Mazeh, T., \& Zucker, S. 2005, MNRAS, 356, 1466 